\title{
Rethinking the discovery function of proof within the context of proofs and
} refutations

\author{
Kotaro Komatsu ${ }^{1}$, Yosuke Tsujiyama ${ }^{2}$ and Aruta Sakamaki ${ }^{3}$
}

${ }^{1}$ Shinshu University, Japan; ${ }^{2}$ Keiai University, Japan; ${ }^{3}$ Nagano Junior High School Attached to the Faculty of Education of Shinshu University, Japan

\begin{abstract}
Proof and proving are important components of school mathematics and have multiple functions in mathematical practice. Among these functions of proof, this paper focuses on the discovery function that refers to an invention of a new statement or conjecture by reflecting on or utilizing a constructed proof. Based on two cases in which eighth and ninth graders engaged in proofs and refutations, we demonstrate that facing a counterexample of a primitive statement can become a starting point of students' activity for discovery, and that a proof of the primitive statement can function as a useful tool for inventing a new conjecture that holds for the counterexample. An implication for developing tasks by which students can experience this discovery function is mentioned.
\end{abstract}

Keywords: Proof and proving; discovery function; refutation; counterexample

- This is an Accepted Manuscript of an article published by Taylor \& Francis in International Journal of Mathematical Education in Science and Technology on October 2014. DOI: 10.1080/0020739X.2014.902135 


\section{Rethinking the discovery function of proof within the context of proofs and refutations}

\section{Introduction}

Proof and proving are characteristics of the discipline of mathematics and play multiple roles in mathematical practice. In addition to verifying the truth of a statement, De Villiers [1] points out other functions of proof such as explanation, systematisation, discovery, and communication. Hanna and Jahnke [2] add three functions: construction of an empirical theory, exploration of the meaning of a definition or the consequences of an assumption, and incorporation of a well-known fact into a new framework and thus viewing it from a fresh perspective. Recently, Hanna and Barbeau [3] argue that proofs can embody methods, tools, strategies and concepts for solving problems.

Among these multiple functions of proof, this paper focuses on the discovery function that indicates 'the discovery or invention of new results' [1, p.18]. This function is important in school mathematics because it has a potential to improve an issue of learning of proof and proving. In school mathematics, it is typical that a statement is given by teachers or textbooks and the truth of the statement is obvious without proving it. This kind of learning may cause many students to feel that proof and proving are ritualistic $[4,5]$. In contrast, the discovery function of proof allows students to engage in a productive activity where they utilise a proof to generate a new result, by themselves, that is often surprising. Therefore, it is expected that the discovery function of proof will enable students to regard proof and proving as a productive tool for inventing new results, rather than as a ritual in which they verify a statement whose truth is already obvious. 
Although mathematics educators have examined the meanings of the discovery function of proof in different settings and examples, this paper addresses the process of proofs and refutations described by Lakatos [6]. His research is well known in our research community, but there is no mathematics education literature that illustrates the discovery function of proof in this process (see Sections 2.1 and 2.2). Lakatos wrote his book Proofs and Refutations to demonstrate that, in informal and quasi-empirical mathematics, mathematical knowledge grew through continuous interaction between conjectures, proofs, and refutations. Therefore, if we can illustrate the discovery function of proof in the context of proofs and refutation using a school mathematics setting, it should become possible to provide students with an opportunity in which they can experience an authentic process that mirrors such mathematical growth.

Consequently, this paper aims to illustrate how proofs can enable students to discover new results in their process of proofs and refutations, with two examples in which junior high school students participated.

\section{The meanings of the discovery function of proof}

\subsection{The discovery function of proof}

When De Villiers refers to the discovery function of proof, he is mainly considering 'deductive discovery via deductive generalization' [1, p.22]; he has illustrated this with several examples in many papers. In order to summarise his point, we illustrate this function with an example that is slightly different from one of his: if we connect the midpoints of each segment in a parallelogram, then we can make a parallelogram.

In order to prove this statement, let $\mathrm{P}, \mathrm{Q}, \mathrm{R}$, and $\mathrm{S}$ be the midpoints of sides $\mathrm{AB}$, $\mathrm{BC}, \mathrm{CD}$, and $\mathrm{DA}$ of parallelogram $\mathrm{ABCD}$, respectively. From the midpoint theorem in triangles $\mathrm{ABD}$ and $\mathrm{BCD}$, diagonal $\mathrm{BD}$ is parallel to side $\mathrm{PS}$, and this diagonal is also 
parallel to side QR. This implies that the two sides PS and QR are parallel. Similar consideration regarding diagonal AC leads to deducing that two sides PQ and SR are parallel. Therefore, quadrilateral PQRS is a parallelogram because it satisfies a condition for parallelograms.

Then, reflecting on this proof shows that it does not use a supposition of the above statement that quadrilateral $\mathrm{ABCD}$ is a parallelogram. Hence, it is possible to generalise the above statement such that even if parallelogram ABCD is replaced with quadrilateral $\mathrm{ABCD}$, quadrilateral $\mathrm{PQRS}$ is still a parallelogram (Figure 1).

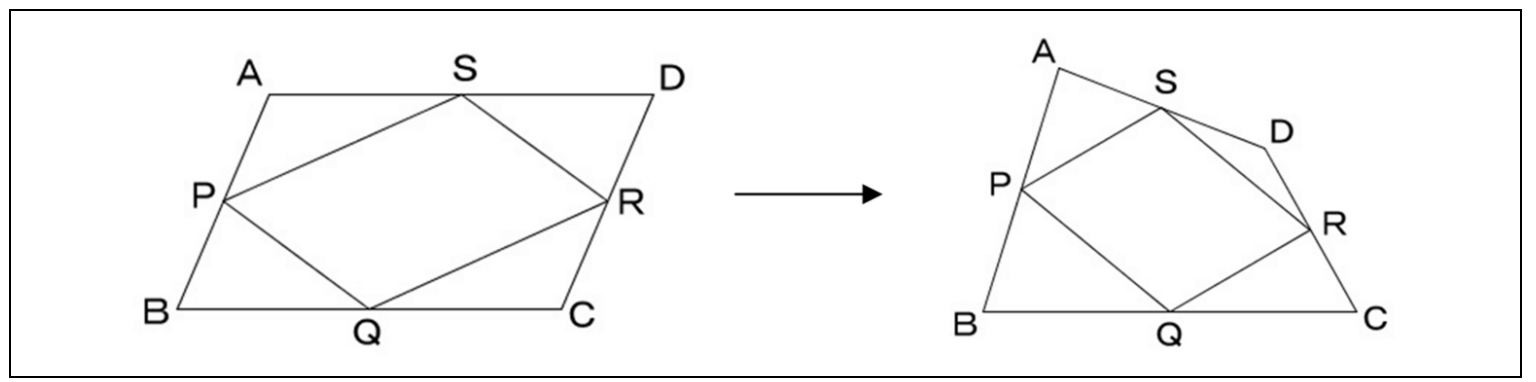

Figure 1. Deductive discovery via deductive generalisation.

When constructing a proof of a statement, a certain supposition of the statement may not be used in the proof. Deductive discovery via deductive guessing refers to inventing a more general statement by finding such supposition and removing it from the statement. This value of proof is discussed by Sugiyama [7] as well.

As Hemmi and Löfwall [8] point out, because there are some misunderstandings regarding the meaning of the discovery function of proof in existing studies, it is important to clarify this meaning. For example, although Knuth [9] refers to the discovery function of proof, he does not consider proof as a method for inventing a new result. Rather, he thinks that such new result is discovered through induction or experiments, and that the discovery function of proof indicates to justifying that the discovered result is true. This interpretation is relevant to the verification function of 
proof, rather than the discovery function, in classification by De Villiers [1]. Ding and Jones [10] attempt to investigate instructional strategies that enable students to see and appreciate the discovery function of proof in geometry. However, they do not mention prior research about this function (for example, [1]), and they consider discovery of properties that are necessary for constructing a proof, rather than discovery of a new result by reflecting on a constructed proof.

De Villiers regards the meaning of the discovery function of proof as 'the discovery or invention of new results' $[1, \mathrm{p} .18]$ and mathematics educators have defined the meaning of these new results diversely. In the above parallelogram example, the discovered result is a statement or conjecture. On the other hand, De Villiers states that 'with the discovery function, it also means that a proof can reveal new, powerful methods of solving problems and creating new theories' $[11$, p.3]. Furthermore, Miyazaki states that 'the results include propositions, proofs, assumptions, concepts, counterexamples, definitions' $[12, \mathrm{p} .2]$. These studies address a global context in which students create new theories or refine existing concepts. Proof and proving in school mathematics, however, are learnt in a more localised context where students only prove statements and sometimes make conjectures, and they do not experience such creation of new theories. Therefore, in order to actualise the discovery function of proof in regular classrooms, this paper limits the meaning of new invented results to statements and conjectures.

In addition, there are differences in the existing literature regarding how new results are invented. The above illustration is an example of 'a discovery made after reflecting on a recently constructed proof' $[11, \mathrm{p} .3]$. On the other hand, De Villiers states differently that 'it (discovery function of proof) also more broadly refers to situations where new results are discovered in a purely logical way by the application of 
known theorems or algorithms without resorting to any experimentation, construction or measurement' [11, p.3]. However, the latter citation is related to the function of deductive reasoning rather than a function of proof. Therefore, this paper restricts methods for inventing a new result to reflecting on or utilising a constructed proof.

In summary, this paper defines the discovery function of proof as an invention of a new statement or conjecture by reflecting on or utilising a constructed proof. This definition does not intend to ignore the diverse meanings of the discovery function discussed above. Rather, this paper limits the meaning of this function in order to make our focus more clear.

\subsection{Illustrations of the discovery function of proof in existing studies}

Even if the meaning of the discovery function of proof is limited as stated above, four types of discovery function, other than deductive discovery via deductive generalisation, have been discussed in existing studies. The first is specialisation rather than generalisation. Though De Villiers [13] illustrates this type with an isosceles trapezoid, we continue the example from the previous section. The proof used the midpoint theorem, which also claims that sides PS and QR are equal to a half of diagonal BD, and that sides PQ and SR are equal to a half of diagonal AC. From this, it can be said that these four sides are equal to each other if the two diagonals are equal. This leads to a statement, 'if we connect the midpoints of each segment in a quadrilateral whose two diagonals are equal, then we can make a rhombus', which is a special case of the statement generalised in the previous section (Figure 2). 

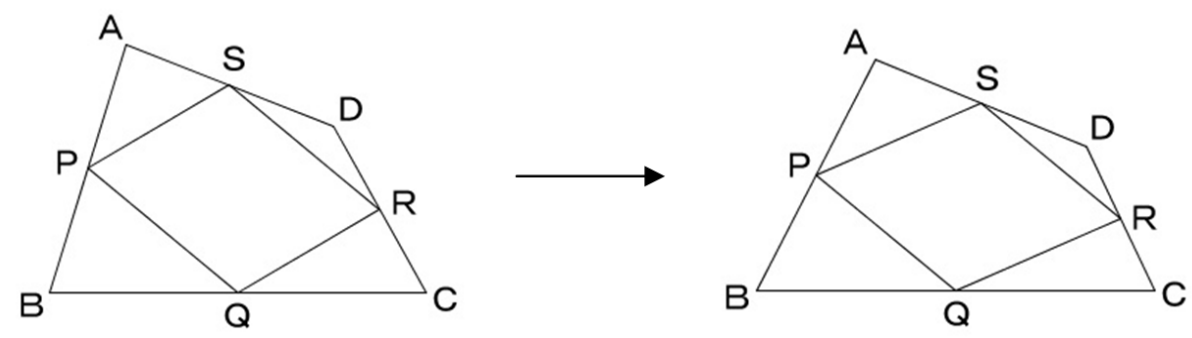

Figure 2. Specialisation.

The second is relevant to an invention of a new statement by analysing some facts obtained in the middle of a constructed proof. De Villiers $[14,15]$ illustrates this with a statement that the three medians in a triangle are concurrent. Let $\mathrm{D}$ and $\mathrm{F}$ be the midpoints of sides $\mathrm{AB}$ and $\mathrm{CA}$, respectively, $\mathrm{O}$ be the point of intersection between medians $\mathrm{CD}$ and $\mathrm{BF}$, and $\mathrm{E}$ be the point of intersection between line $\mathrm{AO}$ and side $\mathrm{BC}$. In order to prove the above statement, it is enough to show that point $\mathrm{E}$ is the midpoint of side $\mathrm{BC}$. Now, $\mathrm{BE} / \mathrm{EC}=\Delta \mathrm{ABO} / \triangle \mathrm{ACO}, \mathrm{CF} / \mathrm{FA}=\Delta \mathrm{BCO} / \Delta \mathrm{ABO}$, and $\mathrm{AD} / \mathrm{DB}=$ $\triangle \mathrm{ACO} / \triangle \mathrm{BCO}$, irrespective of the positions of points $\mathrm{D}, \mathrm{E}$, and $\mathrm{F}$. Since segment $\mathrm{AD}$ is equal to segment $\mathrm{DB}$, the areas of triangles $\mathrm{ACO}$ and $\mathrm{BCO}$ are equal. Similarly, the areas of triangles $\mathrm{BCO}$ and $\mathrm{ABO}$ are equal. Therefore, it is proved that the areas of triangles $\mathrm{ACO}$ and $\mathrm{ABO}$ are equal and thus segment $\mathrm{BE}$ is equal to segment $\mathrm{EC}$. Now, an analysis of the above three fractional expressions leads to finding that the multiplication of these expressions results in the reduction of the right-hand side to 1 . That is, $(\mathrm{BE} / \mathrm{EC}) \times(\mathrm{CF} / \mathrm{FA}) \times(\mathrm{AD} / \mathrm{DB})=1$, which implies Ceva's theorem (Figure 3$)$. 

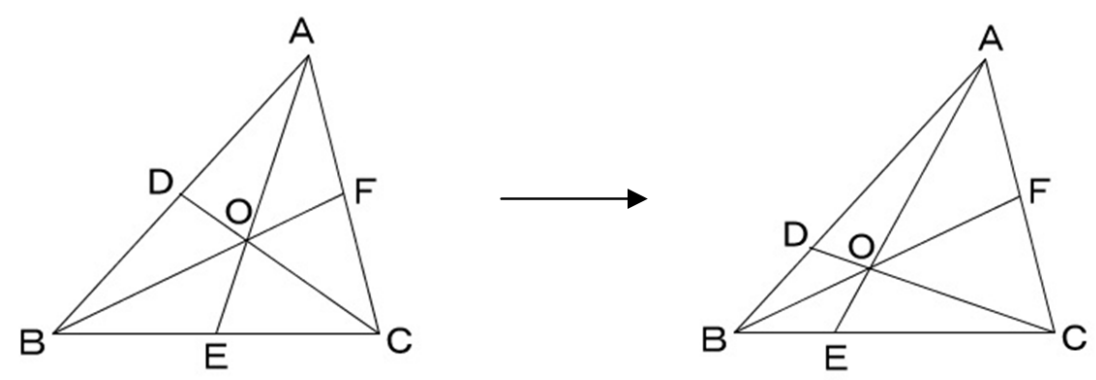

Figure 3. Invention of Ceva's theorem.

The third illustration refers to discovery of a new statement by reflecting on the result obtained at the end of a proof $[11,16]$. For example, in order to prove that the sum of a two-digit integer and the integer whose digits are the reverse of the order of the original integer's digits is always divisible by 11 , students will represent the two numbers as $10 a+b$ and $10 b+a$, and calculate the sum of the two numbers as $11(a+b)$. Examining this expression, they could notice that the other factor is the sum of the digits of the original number (for an example in geometry, see [17]).

Finally, the fourth type is related to changing a supposition of a statement (often generalising) and utilising its proof to find a sufficient supposition. For example, students in an experiment by Miyazaki [12] tackled the following problems (Figure 4):

Problem 1: Place an arbitrary point $\mathrm{P}$ on diagonal BD of rectangle $\mathrm{ABCD}$. Draw a parallel line to segment $\mathrm{AB}$ through $\mathrm{P}$, and let the line intersect with segment $\mathrm{DA}$ at $\mathrm{E}$ and segment $\mathrm{BC}$ at $\mathrm{F}$, respectively. Draw another parallel line to segment $\mathrm{BC}$ through $\mathrm{P}$. Let the line intersect with segment $\mathrm{AB}$ at $\mathrm{G}$ and segment $\mathrm{CD}$ at $\mathrm{H}$, respectively. Prove that the area of quadrilateral AEPG is equal to the area of quadrilateral CFPH.

Problem 2: Draw a segment BD in quadrilateral $\mathrm{ABCD}$, and place an arbitrary point $\mathrm{P}$ on the segment. Draw a parallel line to segment $\mathrm{AB}$ through $\mathrm{P}$, and let the line intersect with segment $\mathrm{DA}$ at $\mathrm{E}$ and segment $\mathrm{BC}$ at $\mathrm{F}$, respectively. Draw another parallel line to segment $\mathrm{BC}$ through $\mathrm{P}$. Let the line intersect with segment $\mathrm{AB}$ at $\mathrm{G}$ and segment $\mathrm{CD}$ at $\mathrm{H}$, respectively. What conditions of quadrilateral 
$\mathrm{ABCD}$ make the area of quadrilateral AEPG equal to the area of quadrilateral CFPH? Prove it.
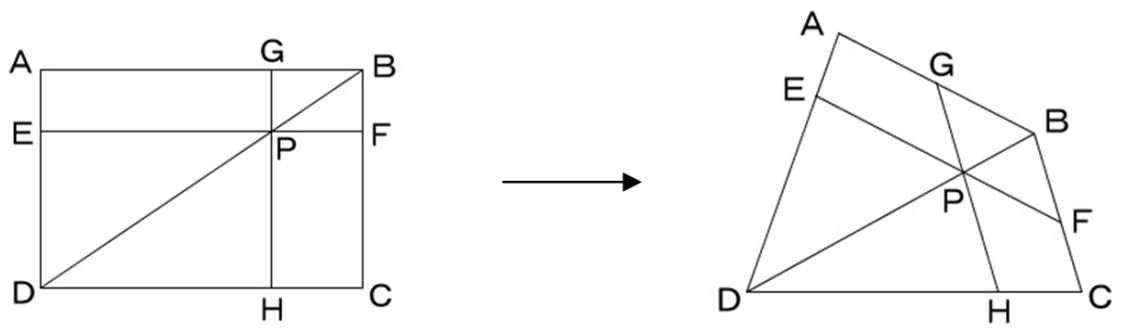

Figure 4. Changing a supposition of a statement.

While solving the first problem, one of the students showed that triangle EDP was congruent to triangle HPD and that the areas of these two triangles were therefore equal. In the beginning of the second problem, she used dynamic geometry software and thought it sufficient to suppose that the area of triangle ABD was equal to the area of triangle CDB. However, she noticed that this supposition did not guarantee the congruence of triangles EDP and HPD used in her previous proof. Therefore, she changed this supposition to the congruence of triangles $\mathrm{ABD}$ and $\mathrm{CDB}$ in order to directly utilise the congruence of triangles EDP and HPD.

In the first, second, and third illustrations, as well as the illustration shown in Section 2.1, discovering a new statement was initiated by reflecting on a constructed proof. On the other hand, a starting point for discovery in the fourth illustration consisted of changing a supposition of a statement and using the constructed proof to find a sufficient supposition.

In contrast to these illustrations, facing a counterexample that refutes a statement has a potential to become an alternative starting point for discovery. In fact, Lakatos [6] described the process in which an imaginary teacher and students confronted a variety 
of counterexamples after proving a primitive conjecture. Then, based on this and other proofs, they restricted the conjecture so that these counterexamples could be excluded, and invented more general conjectures that were true even for these counterexamples. Some of the studies reviewed in the above refer to Lakatos's research in brief (for example, [12]); nevertheless, they do not describe a process in which facing a counterexample leads to discovering a new statement. Therefore, as a starting point for discovery, this paper addresses a case in which students are confronted with a counterexample of a statement, and demonstrates how a constructed proof can function to invent a statement that holds for such counterexample.

\section{Background of examples}

This paper illustrates the discovery function of proof with two cases in the context of proofs and refutations. The first example is taken from a larger study concerning proof and proving with manipulative objects [18]. A pair of Japanese ninth graders (14-15 years old) took part in an experiment conducted by the first author. The second case is derived from a classroom teaching experiment conducted as part of another larger study that aims to develop a set of tasks and associated teachers' guidance that can prompt students to engage in a process of proofs and refutations [19]. The third author conducted this experiment with Japanese eighth graders (13-14 years old). We were all involved in the design of the lessons, and the first author observed all the lessons.

In Japan, eighth and ninth graders learn to prove geometric statements related to various properties of triangles, quadrilaterals, and circles, using conditions for congruent or similar triangles (for more details about proof and proving in the Japanese national curriculum and textbooks, see [20-22]). Because of the difference between the curriculums of their schools, the students in the first case had not yet learnt counterexamples; the students in the second had already known counterexamples. 
All of the experiment and lessons were recorded and transcribed. In addition to these transcripts, data for analysis included what the students wrote on their worksheets, how they utilised manipulatives in the first case, and field notes taken during the lessons in the second example. These data were analysed with a focus on the students' behaviour after the proof construction, in particular, how the students utilised their proofs to invent conjectures that held for counterexamples. We translated the problem sentences, the students' words and proofs from Japanese to English. All of the students' names are pseudonyms.

\section{The first case}

The participants of the first example were Sakura and Yuna. Procedures for choosing them were as follows. All of the ninth graders in their school completed a questionnaire presented by the first author. This investigated their basic knowledge and skill regarding proof. Then, among the students who obtained good results in the questionnaire, Sakura and Yuna were chosen as the participants because, according to their teacher, they had a good rapport and were therefore expected to give rich utterance data for analysing their thinking. These procedures indicate that the two students were strong in proof and proving.

Sakura and Yuna tackled the following problems for about 55 minutes. Because this experiment was conducted as a part of the research relating to proving with manipulatives, they were required to use manipulatives of equilateral triangles made of translucent sheets. In contrast to existing studies on the discovery function of proof and to the second example shown below, it was a characteristic of this first case that the students made a conjecture by themselves. 
Problem 1: We have two equilateral triangles, and place the smaller triangle on top of the bigger one as shown in Figure 5-a. If we join each vertex of the two triangles by a segment, we obtain two segments that are equal in length (thick lines in Figure 5-b). When we rotate one of the two triangles around point $\mathrm{O}$, how will the length relation of these two segments change?

Problem 2: Prove your conjecture in Problem 1.

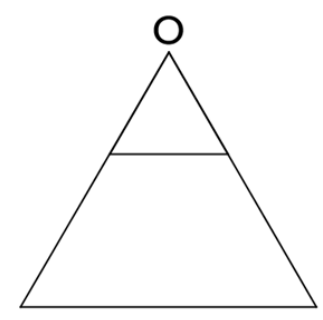

a

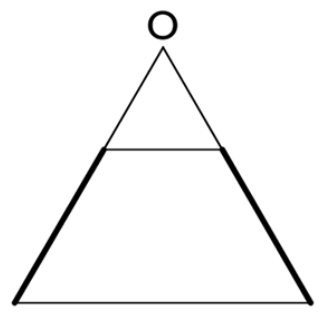

b

Figure 5. Diagrams attached to the problems in the first episode.

\subsection{Primitive conjecture and its proof}

The meaning of these problems was ambiguous for Sakura and Yuna. In particular, they were confused as to which parts 'two segments' (thick lines in Figure 5-b) referred to when rotating one of the two equilateral triangles. In order to solve their confusion, the interviewer used manipulatives to show the meaning of the two segments in a case where the two triangles overlapped each other (hereafter, called the overlapping case).

This intervention had a significant influence on the students, and they consistently examined only the overlapping case. Yuna first conjectured "(are the two segments) always equal in length?", and Sakura agreed with her conjecture. However, they were not able to construct a proof for their conjecture. Therefore, the interviewer advised them to focus on triangles that included the two segments, and this advice enabled them to construct their proof as follows (Figure 6): 
Proof by Sakura and Yuna:

In $\triangle \mathrm{OAB}$ and $\triangle \mathrm{OBD}$

Since $\triangle \mathrm{OAB}$ is an equilateral triangle, $\mathrm{OA}=\mathrm{OB} \ldots$ (1)

Since $\triangle \mathrm{OCD}$ is an equilateral triangle, $\mathrm{CO}=\mathrm{DO} \ldots$ (2)

$\angle \mathrm{AOC}=\angle \mathrm{AOB}-\angle \mathrm{BOC}$

$\angle \mathrm{BOD}=\angle \mathrm{COD}-\angle \mathrm{BOC}$

Therefore, $\angle \mathrm{AOC}=\angle \mathrm{BOD}$

From (1)-(3), since two pairs of sides and the included angles are equal, $\triangle \mathrm{OAC}$ $\equiv \Delta \mathrm{OBD}$

Since corresponding sides of congruent figures are equal, $\mathrm{AC}=\mathrm{BD}$

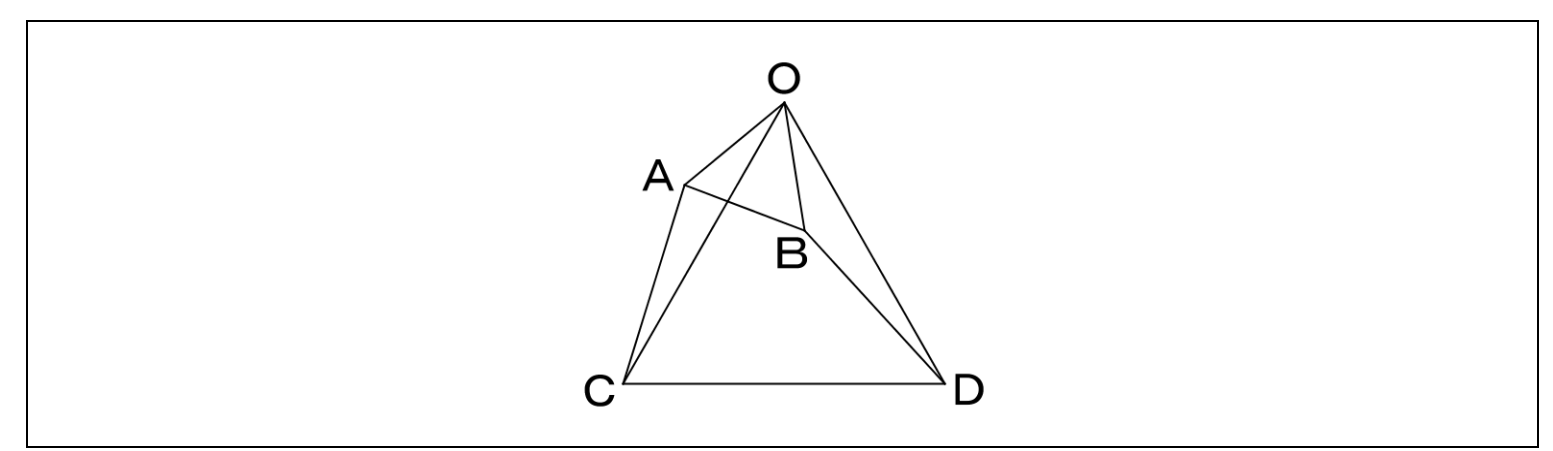

Figure 6. A diagram in the students' proof.

\subsection{Counterexample and generalisation}

After completing their proof, Sakura and Yuna switched to a case in which two equilateral triangles did not overlap (non-overlapping case):

609. Sakura: (The two segments are) equal even if we rotate continuously. (She rotated the manipulative of the smaller triangle to move from an overlapping case to a non-overlapping one.)

612. Yuna: It will be, for sure.

615. Sakura: Wait, how about the case where this (smaller triangle) runs off (bigger one)?

616. Yuna: What?

617. Sakura: What will happen after this? (She considered the two segments as thick lines in Figure 7-a.) Wait, wait. What? 
618. Yuna: We have to rethink this.

635. Sakura: (The two segments are) not absolutely equal.

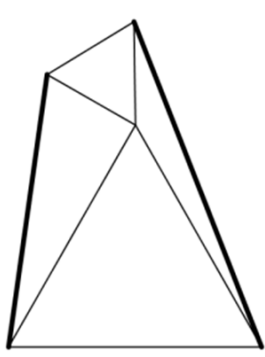

a

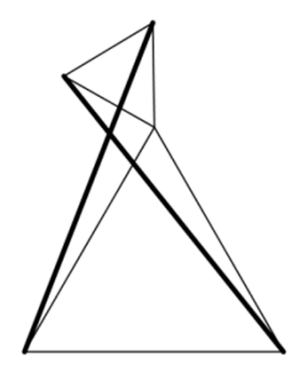

b

Figure 7. A non-overlapping case.

In this dialogue, although the students first conjectured that the two segments were still equal even in the non-overlapping case (609. Sakura and 612. Yuna), they then regarded the non-overlapping case as a counterexample of their conjecture (618. Yuna and 635. Sakura). Of course, if one appropriately grasps the correspondence between the vertices of the two equilateral triangles, the two segments stay equal in length (Figure. 7-b). However, Sakura and Yuna considered the two segments as shown in Figure 7-a, and this resulted in their conclusion that this non-overlapping case became a counterexample of their conjecture. Then, the students restricted their conjecture to only the overlapping case.

Later, Sakura and Yuna revisited the non-overlapping case, and Yuna set the manipulatives as shown in Figure 8-a. In contrast to their previous consideration about the non-overlapping case (Figure 7-a), Sakura grasped the meaning of the two segments as thick lines in Figure 8-a. However, at this point, Yuna still believed that the two segments were not equal, as they had thought before, stating "there seems to be no relationship". 


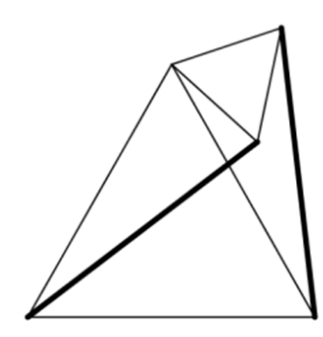

a

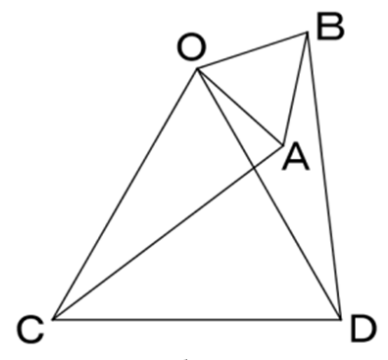

b

Figure 8. A non-overlapping case revisited.

Next, based on their proof for the overlapping case, Sakura labelled each vertex, A to D, and drew segments AC and BD (Figure 8-b). They then proceeded as follows:

801. Sakura: This triangle (she pointed to triangle OBD in the diagram).

802. Yuna: This (triangle OBD).

803. Sakura: And this (triangle OAC).

806. Yuna: It (these triangles are congruent) is still true, isn't it?

807. Sakura: This means, is it (the two segments are equal in length) true in any case?

This interaction demonstrated that the students reflected on and utilised a part of their previous proof, namely the congruence of triangles OAC and OBD, to invent a more general conjecture, $\mathrm{AC}=\mathrm{BD}$ in any case, including the counterexample. To be more exact, they were able to reconsider their initial conjecture, which had been restricted to only the overlapping case, as a more general one that held even in the nonoverlapping case.

There may be an objection that Sakura and Yuna were able to generalise their conjecture because their interpretation of the two segments changed from Figures 7-a to 8 -a, and that their proof did not contribute to this change. However, when they reconsidered the two segments as in Figure 8-a, they still believed that their conjecture failed in the non-overlapping case; as Yuna said "there seems to be no relationship". 
After that, they found that the two segments were equal even in the non-overlapping case because a part of their proof (the congruence of triangles OAC and OBD) seemed to be applicable to this case. Thus, for the students, their proof functioned as a useful tool for inventing a more general conjecture in that they could generalise their initial conjecture to both the overlapping and non-overlapping cases by reflecting on and utilising this proof.

After that, Sakura and Yuna examined whether the triangles OAC and OBD were actually congruent. They first thought that they could deduce this congruence from $\mathrm{OA}=\mathrm{OB}, \mathrm{OC}=\mathrm{OD}$ and $\angle \mathrm{AOC}=\angle \mathrm{BOD}=60^{\circ}-\angle \mathrm{AOD}$. Then, the interviewer asked them to re-examine the included angles, and Yuna noticed that these degrees were not $60^{\circ}-\angle \mathrm{AOD}$, but actually $60^{\circ}+\angle \mathrm{AOD}$.

\section{The second case}

\subsection{Primitive statement and its proof}

The second example is derived from a classroom teaching experiment that consisted of two lessons with 36 eighth graders (50 minutes per lesson). The average of their mathematical abilities was above standard, and they dealt with the following problem:

Problem: As shown in Figure 9, we draw line $l$ that passes point A of square $\mathrm{ABCD}$, and perpendicular lines $\mathrm{BP}$ and $\mathrm{DQ}$ to line $l$ from points $\mathrm{B}$ and $\mathrm{D}$, respectively. Prove that $\mathrm{PQ}=\mathrm{DQ}-\mathrm{BP}$. 


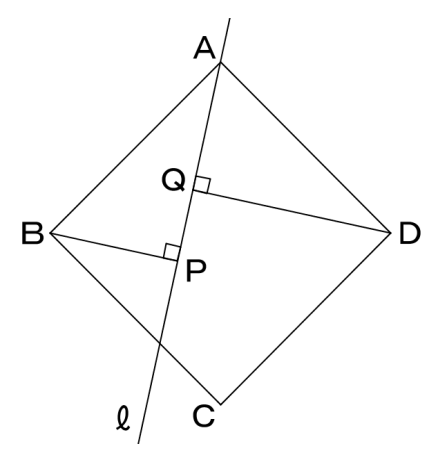

Figure 9. A diagram attached to the problem in the second example.

At the start of the first lesson, the teacher asked his students their plans for solving this problem. Rie and Miho proposed to show the congruence of triangles ABP and DAQ, and Tetsuya mentioned that the two triangles were right triangles. In addition, Ryo stated that showing this congruence led to deducing $\mathrm{AQ}=\mathrm{BP}$ and $\mathrm{AP}=\mathrm{QD}$.

After sharing these ideas, the students tackled this problem individually, and then the teacher had Emi write her proof on the blackboard. Her proof was examined in a classroom discussion, and the part which showed $\angle \mathrm{BAP}=\angle \mathrm{DAQ}$ was complicated for other students. Therefore, the teacher had Mai give her complementary explanation with a different expression.

Proof by Emi and Mai:

In $\triangle \mathrm{ABP}$ and $\triangle \mathrm{DAQ}$,

From the supposition, $\angle \mathrm{APB}=\angle \mathrm{DQA}=90^{\circ}$

Since quadrilateral $\mathrm{ABCD}$ is a square, $\mathrm{AB}=\mathrm{DA}$

Let $\angle \mathrm{BAP}=\mathrm{a}$

Since the sum of the interior angles of triangle ABP is 180 degrees, $\angle \mathrm{ABP}=180^{\circ}$

$-\angle \mathrm{APB}-\angle \mathrm{BAP}=90^{\circ}-\mathrm{a}$

Since an interior angle of a square is 90 degrees, $\angle \mathrm{DAQ}=90^{\circ}-\angle \mathrm{BAP}=90^{\circ}-\mathrm{a}$ Therefore, $\angle \mathrm{ABP}=\angle \mathrm{DAQ}$

Thus, since the hypotenuses and a pair of corresponding angles in right triangles are equal, $\triangle \mathrm{ABP} \equiv \triangle \mathrm{DAQ}$

Since the corresponding sides of congruent figures are equal, $\mathrm{AP}=\mathrm{DQ}$ and $\mathrm{BP}=$ AQ 
Therefore, $\mathrm{PQ}=\mathrm{AP}-\mathrm{AQ}=\mathrm{DQ}-\mathrm{BP}$

\subsection{Counterexamples and new statements}

After this proof, the teacher asked his students "now, we drew line $l$ which passed point A like this (Figure 9), but when the place of this line $l$ is different from here (Figure 9), is it possible to say that this (PQ = DQ - BP) is true?". A few students responded "maybe impossible". Then, the teacher told his students "draw various lines, $l$, which pass point A and investigate by drawing your own diagrams". The first lesson finished when the students drew diagrams individually on their worksheets.

Analysing their worksheets after the lesson, we found that many students drew diagrams similar to those shown in Figures 10 -a and b (these figures are the students' actual drawings). In the case of Figure 10-a, the students wrote, "segment BP becomes longer than segment DQ" or "DQ - BP becomes negative". For Figure 10-b, they wrote, “segment PQ is longer than segments DQ and BP" or "(DQ - BP) becomes negative as well'". Their worksheets evidenced that they grasped these cases as counterexamples that refuted the statement in the original problem $(P Q=D Q-B P)$.

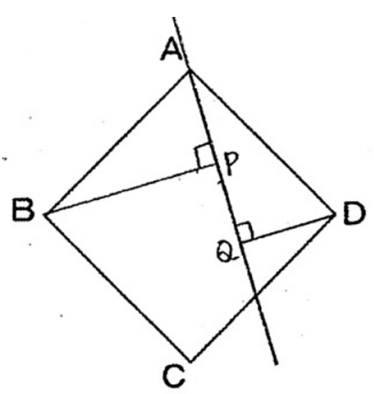

a

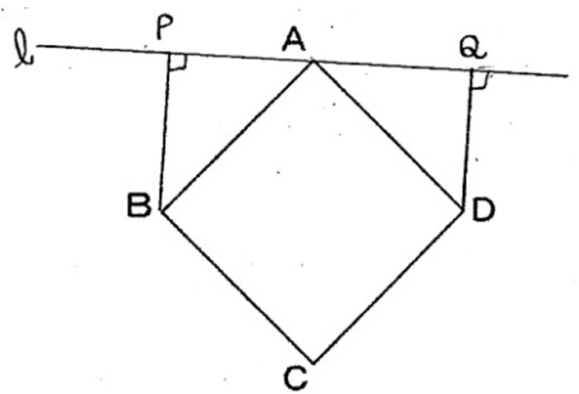

b

Figure 10. Counterexamples drawn by the students. 
In the second lesson, the students investigated what relationships between PQ, $\mathrm{DQ}$, and BP held in the cases as Figures $10-\mathrm{a}$ and $\mathrm{b}$. At this point, the teacher told the students that they were allowed to utilise the previous proof by Emi and Mai.

After the students investigated the problem individually, the teacher had Manabu and Ken write their ideas on the blackboard. Regarding the case represented by Figure 10-b, Manabu wrote, "I prove $\triangle \mathrm{ABP} \equiv \triangle \mathrm{DAQ}$ as we did in the last lesson, and from $\mathrm{PQ}$ $=\mathrm{AQ}+\mathrm{PA}$, it should be true that $\mathrm{PQ}=\mathrm{BP}+\mathrm{QD}$ ”. Ken described his idea for Figure 10 -a as "since $\mathrm{PQ}=\mathrm{AQ}-\mathrm{AP}$ is true, a relationship between $\mathrm{PQ}, \mathrm{DQ}$, and $\mathrm{BP}$ is $\mathrm{PQ}=$ BP - DQ". Thus, Manabu invented a new statement, $\mathrm{PQ}=\mathrm{BP}+\mathrm{QD}$, by utilising the congruence of triangles ABP and DAQ that had been deduced by Emi and Mai. Ken also utilised implicitly this congruence to find a new statement, $\mathrm{PQ}=\mathrm{BP}-\mathrm{DQ}$. Therefore, the students were able to reflect on and utilise the previous proof by Emi and Mai to invent these new statements for the cases as Figures $10-a$ and $b$, which had been counterexamples of the statement in the original problem.

Next, the classroom discussion focused on whether the congruence of triangles ABP and DAQ could actually be deduced by the same proof as proposed by Emi and Mai. For example, in the case of Figure 10-b, the teacher asked his students to examine whether the proof by Emi and Mai was directly applicable to this case. Satoshi questioned the part of this proof that said "since an interior angle of a square is 90 degrees, $\angle \mathrm{DAQ}=90^{\circ}-\angle \mathrm{BAP}=90^{\circ}-\mathrm{a}$ ", and stated that "because both angles DAQ and BAP are not inside it (angle BAD), I think it is not true". Then, other students added that it was possible to deduce ' $\angle \mathrm{DAQ}=180^{\circ}-\angle \mathrm{BAP}-\angle \mathrm{BDA}=90^{\circ}-\mathrm{a}^{\prime}$ by using the fact that $\angle \mathrm{PAQ}=180^{\circ}$.

As in the first example, before reflecting on the proof constructed by Emi and Mai, the students in this case were not able to find out the relationships between 
segments PQ, DQ, and BP in the cases shown in Figures 10-a and b. In fact, Yuko wrote in her worksheet that "I had thought PQ $=\mathrm{DQ}-\mathrm{BP}$ (in the case of Figure 10-a) similar (to the case shown in Figure 9), because the right and left were only reversed, but $I$ found ( $P Q=D Q-B P$ was) not true through copying (the previous) proof'. Toru also wrote that "in the process of making proofs, I gradually understood that I could represent the relationships between PQ, DQ, and BP by using + and -" (emphases by the authors). Therefore, the proof functioned as a useful tool for inventing new statements in that the students were able to find the relationships between the three segments by reflecting on and utilising this proof.

\section{Discussion and conclusion}

This paper aimed to illustrate the discovery function of proof within the context of proofs and refutations [6] that existing studies regarding this function have not addressed explicitly. Though there have been diverse meanings related to this function in the existing studies, this paper defined it as an invention of a new statement or conjecture through reflecting on or utilizing a constructed proof. A characteristic of the discovery function of proof in this paper is in the starting point for discovery. In previous research, such starting points have been limited to reflecting on a constructed proof (for example, [1]) or to changing a supposition of a statement [12]. In contrast to these studies, the two examples referenced in this paper demonstrated that facing counterexamples of primitive statements encouraged students to investigate discovery, and that constructed proofs functioned as a useful tool for inventing conjectures that held for these counterexamples.

The cases in this paper had two additional characteristics compared to the existing studies relating to the discovery function of proof. First, although the illustrations in these studies considered a situation in which primitive statements were 
given by textbooks or teachers, the students in the first example of this paper made a conjecture by themselves. When conjecturing by themselves, students may consider implicitly only a part of the whole situation of a problem, and they may make a conjecture that holds for only the part. Therefore, even after proving the conjecture, students may find a counterexample of the conjecture by considering other cases, and the constructed proof may become an effective tool for exploring a new conjecture. In fact, in the beginning of the first case, Sakura and Yuna examined only the overlapping case, and encountered a counterexample of their conjecture after constructing its proof. Then, by utilising the proof, they were able to generate a more general conjecture that held even for the counterexample (for an arithmetic example at the primary school level, see [23]).

Second, the problem in the second episode was presented with a diagram, and the problem sentence did not express all of the suppositions explicitly; a specific supposition, namely the position of line $l$, was implicitly embedded in the diagram. Although previous studies have dealt with problems whose suppositions are clearly stated in the problem sentences, most proof problems in school geometry include diagrams and some of such problems have implicit suppositions in the diagrams. Hence, transforming the diagram of the original problem may lead to finding a counterexample or non-example, and the proof of the problem may be helpful to investigating new conjectures for the transformed diagrams [19]. In fact, in the second case, Emi and Mai constructed the proof of the original problem, and then the students found out counterexamples by changing the position of line $l$. After that, they could utilise this proof to invent new conjectures that held for these counterexamples.

The discovery function of proof in the first example is valuable from a viewpoint of Proofs and Refutations [6]. Lakatos formulated five heuristic rules as 
normative actions that one should take when facing counterexamples. One of the rules referred to 'increasing content by deductive guessing', which insisted that '(i)f you have counterexamples of any type, try to find, by deductive guessing, a deeper theorem to which they are counterexamples no longer' $[6, \mathrm{p} .76]$. In the first example in this paper, Sakura and Yuna proved their conjecture and were then confronted with its counterexample (the non-overlapping case). Nevertheless, by utilising their proof, they were able to reconsider their conjecture as a deeper one which the non-overlapping case was no longer a counterexample. Thus, the discovery function of proof in this example mirrors a portion of proofs and refutations.

In contrast to the first case, the students in the second example could not invent a general statement that included the three statements. In theory, it is possible to invent such a statement if we represent $P Q=|\overrightarrow{B P}+\overrightarrow{D Q}|[24]$. However, the students in this example had learnt neither vector nor the expression of absolute value. For this reason, it was impossible for them to unify all cases, and the case analysis according to the positions of line $l$ was sufficient for them.

A point in common between the two examples is that the supposition of the primitive statement was not clear enough when the students undertook proving them. Nevertheless, whether the statement was conjectured by the students or given by the teacher, they engaged in the process of constructing its proof, facing counterexamples, and inventing a new statement through reflecting on or utilising the proof. If we examine this process purely logically, the 'proof' was not valid since the supposition of the target statement was not clear and it actually included counterexamples. However, for the students, the meaning of the statement depended on their interpretation and gradually changed as they engaged in the process. Existing studies on the discovery function have mentioned what can be discovered and how (see Sections 2.1 and 2.2), 
but their considerations are based on a mathematically valid proposition and its proof. Considering our cases and the fact that similar situations often happened in mathematical history (for example, mathematicians' efforts from the ancient Greek era and beyond toward Euclid's Elements, see [25]), it is important to extend the meaning of a primitive statement in the study of the discovery function. More concretely, it is valuable to begin with a statement whose meaning is obscure and to provide students with an opportunity in which they explore the statement through proving, such as searching an implicit assumption and examining a possibility to generalize the statement.

The cases in this paper also have an implication for developing tasks related to the discovery function of proof. Hanna and De Villiers point out that 'another underexplored research area encompasses the identification of good problems and the development of effective strategies to help students see and appreciate this "discovery" function of proof' [26, p.10]. Both examples in this paper consisted of specific tasks in which primitive statements were denied when diagrams attached to the tasks were transformed. By utilising the constructed proofs with appropriate modification, the students were able to invent new statements for the cases in which the primitive statements had been invalidated. This paper therefore implies that a certain kind of tasks, in which primitive statements collapse when diagrams attached to the tasks are transformed, has a potential for students to experience the discovery function of proof.

This paper did not aim to exhaust diverse meanings of the discovery function of proof. Rather, we aimed to illustrate this function within a context that existing studies have not addressed, with keeping the meaning 'the discovery or invention of new results' $[1, \mathrm{p} .18]$. Therefore, it is necessary to explore other meanings of this function that have not been addressed or discussed in prior studies or this paper. Furthermore, though the students in both cases were able to utilise their proofs for discovery, they were strong in 
mathematics and still needed teaching intervention. This fact implies that utilising a proof for discovery is difficult for many students in usual classrooms and careful instruction is essential. In the future, it will be necessary to develop effective instructional strategies that can facilitate students to engage in and appreciate the discovery function of proof.

\section{Acknowledgements}

Parts of earlier versions of this paper were presented at the 35th Conference of the International Group for the Psychology of Mathematics Education (Ankara, Turkey, 2011). This study is supported by Grants-in-Aid for Scientific Research [grant number 23330255], [grant number 24243077], [grant number 24730730], [grant number 24830017].

\section{References}

[1] De Villiers, M. The role and function of proof in mathematics. Pythagoras. 1990; 24: $17-24$.

[2] Hanna, G., Jahnke, H. N. Proof and proving. In: A. J. Bishop, M. A. Clements, C. Keitel, J. Kilpatrick, C. Laborde, eds. International handbook of mathematics education. Dordrecht: Kluwer Academic Publishers; 1996. p. 877-908.

[3] Hanna, G., Barbeau, E. Proofs as bearers of mathematical knowledge. ZDM. 2008; 40: 345-353.

[4] Harel, G., Sowder, L. Students' proof schemes: Results from exploratory studies. In: A. H. Schoenfeld, J. Kaput, E. Dubinsky, eds. Research in collegiate mathematics education, Vol. III. Providence, RI: American Mathematical Society; 1998. p. 234-283.

[5] Healy, L., Hoyles, C. A study of proof conception in algebra. J. Res. Math. Educ. 2000; 31: 396-428.

[6] Lakatos, I. Proofs and refutations: The logic of mathematical discovery. Cambridge: Cambridge University Press; 1976. 
[7] Sugiyama, Y. Koritekihoho ni motozuku sansu/sugaku no gakushushido [Mathematics education based on ideas of axiomatic method]. Tokyo: Toyokan Publishing; 1986. Japanese.

[8] Hemmi, K., Löfwall, C. Making the discovery function of proof visible for upper secondary school students. In: M. Pytlak, T. Rowland, E. Swaboda, eds. Proceedings of the Seventh Congress of the European Society for Research in Mathematics Education. Rzeszöw, Poland; 2011. p. 172-181.

[9] Knuth, E. J. Teachers' conceptions of proof in the context of secondary school mathematics. J. Math. Teach. Educ. 2002; 5: 61-88.

[10] Ding, L., Jones, K. Instructional strategies in explicating the discovery function of proof for lower secondary school students. In: F. L. Lin, F. J. Hsieh, G. Hanna, De Villiers, M, eds. Proceedings of the ICMI Study 19 Conference: Proof and Proving in Mathematics Education. Taipei, Taiwan; 2009. Vol. 1, p. 136-141.

[11] De Villiers, M. An illustration of the explanatory and discovery functions of proof. Pythagoras. 2012; 33: Article 193.

[12] Miyazaki, M. What are essential to apply the "discovery" function of proof in lower secondary school mathematics?. In: T. Nakahara, M. Koyama, eds. Proceedings of the 24th Conference of the International Group for the Psychology of Mathematics Education. Hiroshima, Japan; 2000. Vol. 4, p. 1-8.

[13] De Villiers, M. Using dynamic geometry to expand mathematics teachers' understanding of proof. Int. J. Math. Educ. Sci. Technol. 2004; 35: 703-724.

[14] De Villiers, M. An alternative approach to proof in dynamic geometry. In: R. Lehrer, D. Chazan, eds. Designing learning environments for developing understanding of geometry and space. Mahwah, NJ: Lawrence Erlbaum Associates; 1998. p. 369-393.

[15] De Villiers, M. Rethinking proof with the geometer's sketchpad. Emeryville, CA: Key Curriculum Press; 2003.

[16] De Villiers, M. Computer verification vs. algebraic explanation. Pythagoras. 1993; 31: 46.

[17] Chino, K., Fujita, T., Komatsu, K., Makino, T., Miyakawa, T., Miyazaki, M., Mizutani, N., Nakagawa, H., Otsuka, S., Tsujiyama, Y. An assessment framework for students' abilities/competencies in proving. In: Y. Shimizu, Y. Sekiguchi, K. Hino, eds. Proceedings of the 5th East Asia Regional Conference on Mathematics Education. Tokyo, Japan; 2010. Vol. 2, p. 416-423. 
[18] Komatsu, K. How do students generalize a conjecture through proving?: The importance of boundary cases between example and counterexample. In: B. Ubuz, ed. Proceedings of the 35th Conference of the International Group for the Psychology of Mathematics Education. Ankara, Turkey; 2011. Vol. 3, p. 89-96.

[19] Komatsu, K., Tsujiyama, Y. Principles of task design to foster proofs and refutations in mathematical learning: Proof problem with diagram. In: C. Margolinas, ed. Task Design in Mathematics Education: Proceedings of ICMI Study 22. Oxford, United Kingdom; 2013. p. 471-479.

[20] Hoyles, C., Foxman, D., Küchemann, D. A comparative study of geometry curricula. London: Qualifications and Curriculum Authority; 2002.

[21] Miyakawa, T. Proof in geometry: A comparative analysis of French and Japanese textbooks. In: T. Y. Tso, ed. Proceedings of the 36th Conference of the International Group for the Psychology of Mathematics Education. Taipei, Taiwan; 2012. Vol. 3, p. 225-232.

[22] Miyazaki, M., Fujita, T. Proving as an explorative activity in mathematics education. In: B. Sriraman, J. Cai, K. H. Lee, L. Fan, Y. Shimizu, C. S. Lim, K. Subramaniam, eds. The first sourcebook on Asian research in mathematics education: China, Korea, Singapore, Japan, Malaysia and India. Charlotte, NC: Information Age Publishing; in press.

[23] Komatsu, K. Counter-examples for refinement of conjectures and proofs in primary school mathematics. J. Math. Behav. 2010; 29: 1-10.

[24] Shimizu, S. Sansu/sugaku kyoiku ni okeru "mondai" no seikaku ni tsuiteno ichikosatsu: Zoku [Characteristics of "problem" in mathematics education (II)]. Epsilon: Bul. Dept. Math. Educ. Aichi Univ. Educ. 1981; 23: 29-43. Japanese.

[25] Heath, T. L. The thirteen books of Euclid's Elements (second edition) Vol.1: Introduction and Books I, II. (translated from the text of Heiberg). New York: Dover Publications; 1956. (Original work published 1908)

[26] Hanna, G., De Villiers, M. Aspects of proof in mathematics education. In: G. Hanna, M. De Villiers, eds. Proof and proving in mathematics education: The 19th ICMI Study. New York: Springer; 2012, p. 1-10. 